\title{
Condyloma latum mimicking keratoacanthoma in patient with secondary syphilis
}

\author{
S N THAM, C T LEE \\ From the Middle Road Hospital, Singapore
}

SUMMARY A 31 year old man with a condyloma latum simulating a keratoacanthoma at the umbilicus is reported. The umbilicus should be included in the list of known sites of predilection for condylomata lata.

\section{Case report}

A 31 year old Chinese man presented to the dermatological outpatient department of this hospital in January 1986 with a rapidly enlarging umbilical lesion of three weeks' duration. Clinical examination disclosed a slightly tender dome shaped granular tumour, $2.5 \mathrm{~cm}$ diameter, with a central crateriform ulcer overlaid by a purulent discharge (figure 1). General examination showed no abnormality apart from mild diffuse scalp alopecia. A clinical diagnosis of an infected keratoacanthoma was made, with the differential diagnoses of a squamous cell carcinoma or an atypical pyogenic granuloma. Oral ampicillin and cloxacillin were prescribed.

Examination of a biopsy specimen from the lesion showed epidermal hyperkeratosis, papillomatosis, acanthosis, and follicular plugging. The dermis showed dilated capillaries and a dense superficial and deep perivascular infiltrate of plasma cells, lymphocytes, and histiocytes. The finding on histology was consistent with a condyloma latum. The Venereal Disease Research Laboratory (VDRL) slide test, which is routinely offered to patients attending our dermatology outpatient department, was strongly positive. The fluorescent treponemal antibody absorption (FTA-ABS) test performed on the same sample of blood also gave a positive result. A swab taken from the purulent exudate for routine bacteriological culture was sterile.

Further enquiry revealed that the patient had had

Address for reprints: Dr S N Tham, Middle Road Hospital, 250 Middle Road, Singapore 0718.

Accepted for publication 17 January 1987 sexual intercourse with a prostitute about three months before the onset of the lesion, but had not developed any signs of early infectious syphilis after the exposure. The diagnosis was revised to secondary syphilis with umbilical condyloma latum, and the patient was treated with two intramuscular injections of benzathine penicillin 2.4 MIU given a week apart. Review one month after the start of treatment disclosed only slight post inflammatory hyperpigmentation (figure 2). Follow up VDRL tests were reactive at a titre of $1 / 16$ one month later, and $1 / 4$ six months later.

\section{Discussion}

Keratoacanthoma is a benign tumour that occurs more commonly on the face and hands than on

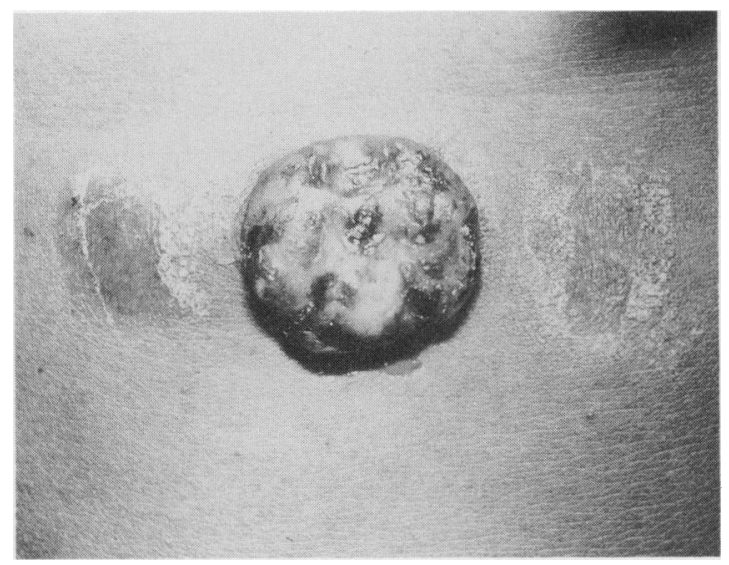

Fig. 1 Dome shaped granulating tumour $2.5 \mathrm{~cm}$ in diameter with a crateriform ulcer and slough on the surface. 


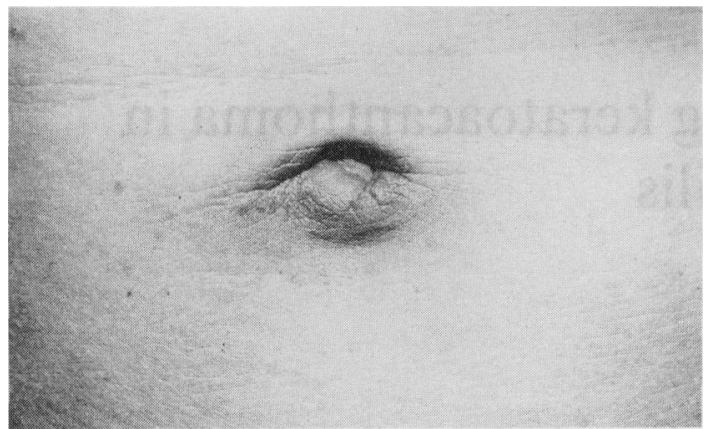

Fig. 2 Slight hyperpigmentation one month after the start of treatment with benzathine penicillin, two $2.4 \mathrm{MIU}$ intramuscular injections given one week apart.

clothed skin. In a study of 250 keratoacanthomas occurring in 238 patients, the umbilicus was not included as a site where keratoacanthomas were usually found. ${ }^{1}$ The diagnosis in our patient was decided from the results of the histological examination and the treponemal serology tests, which excluded the other diagnoses. Condyloma latum of the umbilicus has been reported before. ${ }^{2}$
This case illustrates two important points. Firstly, the umbilicus is a moist and occluded site and should therefore be included in the list of known sites of predilection for condylomata lata. ${ }^{34}$ Secondly, condylomata lata may mimic keratoacanthoma clinically. Biopsy samples should be taken from lesions like keratoacanthomas occurring in unusual sites, and treponemal serology tests should be performed if there is a history of sexual exposure.

We thank Dr KB Lim for reviewing the manuscript.

\section{References}

1 Ghadially FN. Keratoacanthoma. In: Fitzpatrick TB, Eisen AZ Wolf K, Freedberg IM, Austen KF, eds. Dermatology in general practice. 2nd ed. New York: McGraw-Hill, 1979:383-9.

2 Liu TT, Tan M, Goh YS. Condylomata lata of the umbilicus. Singapore Med J 1972;13:321-2.

3 Knox JM, Rudoph AH. Acquired infectious syphilis. In: Holmes KK, Mårdh P-A, Sparling PF, Wiesner PJ, eds. Sexually transmitted diseases. New York: McGraw-Hill, 1984:305-13.

4 Fiumara NJ. Unusual location of condylomata lata. British Journal of Venereal Diseases 1977;53:391-3. 